\title{
On the search of new treatment strategies in patients with affective disorders
}

\author{
Andrea Schmitt $^{1} \cdot$ Peter Falkai $^{1}$
}

Published online: 25 October 2017

(c) Springer-Verlag GmbH Germany 2017

\begin{abstract}
Affective disorders, including major depressive disorder and bipolar disorder, are the most prevalent psychiatric diseases. Major depression has a lifetime prevalence of about $20 \%$, while lifetime prevalence of bipolar disorder is $3 \%$ for all subtypes within the bipolar spectrum [1]. Among all mental illnesses, major depression, bipolar disorder, and schizophrenia cost 207 billion Euro in direct and indirect costs per year (113 billion $€$ for major depression and 94 billion $€$ for schizophrenia and bipolar disorder) [2]. These massive socioeconomic costs extend those for cardiovascular diseases and are expected to rise over the next few decades, with major depression being the leading cause for years lived with disability [3]. All the types of affective disorders display affective and cognitive deficits. Impaired cognitive performance has consistently been reported in major depression and bipolar disorder, mainly in attention, memory, and executive tasks [1]. These cognitive symptoms contribute to loss of social skills and vocational problems. To successfully improve outcomes, novel therapeutic modes of actions are needed in addition to currently available psychopharmacological treatments: antipsychotics, antidepressants, and mood stabilizers, which fall short in averting disabling disease courses in $30-50 \%$ of the patients.

Current pathophysiological concepts in affective disorders, which emphasize the effect of aerobic exercise on adult neurogenesis, hippocampal dysfunction, and volume loss and neurotrophic signalling, have been proposed [1]. Effects of physical exercise on mood symptoms have been
\end{abstract}

Andrea Schmitt

Andrea.Schmitt@med.uni-muenchen.de

1 Department of Psychiatry and Psychotherapy, LudwigMaximilians-University Munich, Nußbaumstr. 7, 80336 Munich, Germany associated with increased production of brain neurotrophic factors (e.g., BDNF) and increased activity and production of neurotransmitters (serotonin, norepinephrine, and dopamine) related to affective disorders. In patients with major depression, improved working memory, psychosocial functioning, and depressive symptoms have been reported after aerobic exercise [4]. Proving the effect of aerobic exercise on remission, it could be shown that of non-remitted patients with major depression, $29.5 \%$ remitted after a 3 months lasting aerobic exercise treatment [5]. A review of effects of aerobic exercise in bipolar disorder revealed that the results in bipolar disorder mirror those from studies in major depression, suggesting that exercise is an innovative treatment option for neurocognitive dysfunction in bipolar disorder [6]. Feasibility and physical adaptations to endurance training have already been shown in patients with severe psychiatric disorders such as chronic schizophrenia [7] and future studies should be extended to patients with affective disorders.

Based on clinical pilot studies and randomized monocentric clinical trials, another novel treatment option, transcranial direct current stimulation (tDCS), has been proposed in patients with major depression. In this issue, Padberg et al. [8] describe a new multicenter trial using advanced tDCS technologies, genetic testing, and functional magnetic resonance imaging. The trial may answer the question whether prefrontal tDCS is a safe and effective antidepressant intervention in patients who have not sufficiently responded to SSRIs. In this issue, Herzog et al. [9] described that a large proportion of patients with major depression is not treated in adherence to current treatment guidelines which propose therapeutic drug monitoring and treatment change after 4-8 weeks in non-responders. In summary, optimized treatment with antidepressants as well as add-on treatment with new treatment strategies such as aerobic exercise [10] and 
tDCS [8] may improve the clinical response in patients with affective disorders.

\section{References}

1. Malchow B, Oertel-Knöchel V, Cahn W, Keller K, Reich-Erkelenz D, Hasan A, Scheewe T, Schmitt A, Falkai P, Kahn RS (2013) The effects of physical exercise in schizophrenia and affective disorders. Eur Arch Psychiatry Clin Neurosci 263(6):451-467

2. Gustavsson A et al (2011) Cost of disorders of the brain in Europe 2010. Eur Neuropsychopharmacol 21(10):718-779

3. Vos T et al (2015) Global, regional, and national incidence, prevalence, and years lived with disability for 301 acute and chronic diseases and injuries in 188 countries, 1990-2013: a systematic analysis for the global burden of disease study 2013. Lancet 386(9995):743-800

4. Greer TL et al (2016) Improvements in psychosocial functioning and health-related quality of life following exercise augmentation in patients with treatment response but nonremitted major depressive disorder: results from the tread study. Depress Anxiety. 33(9):870-881

5. Rethorst CD et al (2017) Prediction of treatment outcomes to exercise in patients with nonremitted major depressive disorder.
Depress Anxiety. https://doi.org/10.1002/da.22670 (Epub ahead of print)

6. Kucyi A, Alsuwaidan MT, Liauw SS, McIntyre RS (2010) Aerobic physical exercise as a possible treatment for neurocognitive dysfunction in bipolar disorder. Postgrad Med 122(6):107-116

7. Keller-Varady K, Hasan A, Schneider-Axmann T, Hillmer-Vogel U, Adomßent B, Wobrock T, Schmitt A, Niklas A, Falkai P, Malchow B (2016) Training in schizophrenia patients and healthy subjects: differences and similarities. Eur Arch Psychiatry Clin Neurosci 266(5):461-473

8. Padberg F, Kumpf U, Mansmann U et al (2017) Prefrontal transcranial direct current stimulation (tDCS) as treatment for major depression: study design and methodology of a multicenter triple blind randomized placebo controlled trial (Depression CD). Eur Arch Psychiatry Clin Neurosci 1-16. doi: 10.1007/ s00406-017-0769-y

9. Herzog DP, Wagner S, Ruckes C et al (2017) Guideline adherence of antidepressant treatment in outpatients with major depressive disorder: a naturalistic study. Eur Arch Psychiatry Clin Neurosci 1-11. doi: 10.1007/s00406-017-0798-6

10. Schmitt A, Falkai P (2017) Aerobic exercise in major psychiatric disorders: promises and challenges. Eur Arch Psychiatry Clin Neurosci 267(2):93-94 